\title{
Thrust and Efficiency of Propulsion by Oscillating Foils
}

\author{
J. Young ${ }^{1}$, J.C.S. Lai ${ }^{1}$, M.Kaya $^{2}$ and I.H. Tuncer ${ }^{2}$ \\ 1 School of Aerospace, Civil and Mechanical Engineering, UNSW@ADFA, \\ Australian Defence Force Academy, Canberra, ACT, 2600, Australia, \\ j.young@adfa.edu.au, j.lai@adfa.edu.au \\ 2 Department of Aerospace Engineering, Middle East Technical University, 06531, \\ Ankara, Turkey, mkaya@ae.metu.edu.tr, tuncer@ae.metu.edu.tr
}

\section{Introduction}

Oscillating foils are being considered for propulsion of submersible vehicles and micro air vehicles (MAVs), at low Reynolds numbers where conventional propulsion techniques become inefficient. Linearized theories and potential flow methods provide good estimates of thrust and propulsive efficiencies at higher Reynolds numbers, but cannot account for the leading-edge flow separation and vortex formation that can occur in viscous flows. A 2D unsteady Navier Stokes solver was used to explore the effect of flow separation on the thrust and efficiency of an airfoil undergoing various combinations of sinusoidal pitching and plunging motions.

The Navier Stokes solver has been validated against a number of flow visualizations for pure plunging motion, and quantitative experimental and computational studies of pure pitching motion, with good results [1]. Here combined pitching and plunging motions are computed, with thrust and efficiency results compared to small-amplitude potential theory (Garrick [2]), largeamplitude unsteady panel method (UPM) potential flow simulations $[1,3]$, and experimental results of Anderson et al [4].

\section{Numerical Method}

The unsteady flow field around the oscillating airfoil is calculated using a 2ndorder accurate unsteady 2D compressible Navier Stokes solver. Motion of the airfoil is introduced by a combination of rigid-body motion and deformation of the grid, resulting in time-dependent grid metrics and Jacobian. Details of the method may be found in Young and Lai [1,3], and Tuncer and Platzer [5]. Versions of the code have been parallelized with OpenMP for use on an Alpha cluster, and Parallel Virtual Machine (PVM) for use on a cluster of Pentiumbased workstations running Linux.

The motion of the airfoil is a combination of plunging, $y=a \sin (\omega t)$ and pitching, $\theta=\theta_{0} \sin (\omega t+\phi)$. Parameters of interest are the reduced frequency 
$k=\omega c / 2 U_{\infty}$, non-dimensionalized plunge amplitude $h=a / c$, pitch amplitude $\theta_{0}$, pivot point of the pitching motion, and the phase angle $\phi(c$ is the airfoil chord and $U_{\infty}$ is the free-stream velocity). The Strouhal number is calculated based on the frequency of motion and the total (peak to peak) excursion $A_{T E}$ of the trailing edge, $S t=\omega A_{T E} / 2 \pi U_{\infty}=k A_{T E} / \pi c$. Outputs include the time-averaged thrust coefficient $\overline{C_{T}}=-1 / T \int_{t}^{t+T} C_{D}(t) d t$, input power coefficient $\overline{C_{P_{i n}}}=-1 / T \int_{t}^{t+T}\left[C_{L}(t) \dot{y}(t) / c+C_{M}(t) \dot{\theta}(t)\right] d t$, and propulsive efficiency, $\eta=\overline{C_{T}} U_{\infty} / \overline{C_{P_{i n}}} . C_{D}, C_{L}$ and $C_{M}$ are the drag, lift and moment coefficients calculated by integrating viscous and pressure forces around the airfoil surface.

Optimization of time-averaged thrust and propulsive efficiency is performed using a gradient-descent based method. The objective function is a linear combination of the thrust coefficient and the efficiency, and the optimization variables are $h, \theta_{0}, k$, and $\phi$. Evaluation of the gradient vector involves unsteady flow computation over a number of motion cycles until periodic behavior is established. Each of the gradient vector components is calculated in parallel. Calculations were typically performed on a $541 \times 61$ grid, with 189 points on the airfoil, a first grid point at $8.75 \times 10^{-6}$ chords from the airfoil surface, and a boundary 20 chords from the airfoil in all directions.

\section{Comparison of Numerical and Experimental Results}

Figs. 1, 2, and 3 show the time-averaged thrust coefficient and the propulsive efficiency vs Strouhal number for three different motions. The parameter $\alpha_{0}=-\tan ^{-1}(2 k h)+\theta_{0}$ is kept constant at $15^{\circ}$ for all cases. Reynolds number $R e=40,000$, and the airfoil is pitching about a point $1 / 3$ chords aft of the leading edge. These conditions were chosen to match those of the experiment performed by Anderson et al [4]. Navier Stokes calculations were performed using fully laminar flow, fully turbulent flow with the Baldwin-Lomax turbulence model, and a transition model using the Spalart-Allmaras turbulence model. Negligible differences in thrust and efficiency were found between the Baldin-Lomax and Spalart-Allmaras models. Fig. 1, for which leading edge separation is minimal across the range of Strouhal number, shows relatively good agreement with the measurements of Anderson et al [4], and little difference between laminar and turbulent flows. Figs. 2 and 3, for which large leading edge separation is apparent at some Strouhal numbers, show lower efficiencies than measured by Anderson et al, and indicate a high degree of sensitivity to the precise details of leading edge vortex formation, dependent on whether the flow is assumed laminar or turbulent. In all three cases the level of agreement with experiment is good for the thrust coefficient, and moderate for the propulsive efficiency although the trends are well predicted. 
It should be noted that small discrepancies in time-varying quantities may become relatively larger errors in the time-averaged quantities.

\section{Optimization of Thrust and Efficiency}

Figs. 1, 2 and 3 taken together show that the propulsive efficiency of an oscillating airfoil is sensitively dependent on the phase angle between pitching and plunging motion. This is in agreement with the findings reported by Isogai et al [6] for $R e=10^{5}$ and Tuncer and Kaya [7], suggesting that high efficiencies and thrusts may be simultaneously achieved by optimizing interaction of the leading edge vortex with the foil motion.

The optimization procedure was checked by allowing the frequency and pitch amplitude to vary while holding other parameters fixed, and optimizing against efficiency. The results are shown in Fig. 2. In both laminar and turbulent cases the optimized solution converges to a point close to the peak efficiency found by a parametric search. In Table 1 the plunge amplitude is held constant at $h=0.75, \alpha_{0}=15^{\circ}$, and the oscillation frequency and pitch amplitude are optimized. The procedure is repeated both with phase angle held constant, and optimized. Laminar and turbulent flows are considered. The table shows that the optimum phase angle between pitch and plunge motion is somewhere between $\phi=75^{\circ}$ to $85^{\circ}$, again in good agreement with Anderson et al [4] and Isogai et al [6].

The effect of leading edge separation on thrust and efficiency may be seen in Figs. 4 and 5. Here numerical particle traces are shown for four points in the motion cycle for turbulent flow at two different Strouhal numbers. Fig. 4 corresponds to the highest efficiency point of Fig. 3 ( $S t=0.184$, $\left.\overline{C_{T}}=0.24, \eta=0.72\right)$, and Fig. 5 is a high thrust, low efficiency case $(S t=$ $\left.0.70, \overline{C_{T}}=1.22, \eta=0.44\right)$. Optimization of the flapping motion for highest efficiency appears to minimize leading edge separation. Optimization for high thrust promotes leading edge separation on the airfoil surface which is tilted upstream, so the low pressure of the separated vortex results in thrust rather than drag. The low thrust, low efficiency cases in Figs. 2 and 3 correspond to leading edge separation on the airfoil surface that is is tilted downstream.

\section{Conclusion}

Simulations of the flow over an oscillating airfoil show good agreement with the experimental results of Anderson et al [4], for a range of different flapping parameters. The propulsive efficiency peaks at a Strouhal number between 0.15-0.3, as found by Anderson et al and other researchers. Gradient-descent based optimization of the phase angle between the pitching and plunging components of the motion shows a phase angle between $\phi=75^{\circ}$ and $85^{\circ}$ for best propulsive efficiency, in agreement with Anderson et al [4] and Isogai et 
al [6]. Highest efficiency propulsion occurs when leading edge separation is avoided or minimized, however thrust is low in this case. High thrust corresponds to long periods of high effective angle of attack, and large separated leading edge vortices.

\section{Acknowledgements}

This work was supported by an award under the Merit Allocation Scheme on the National Facility of the Australian Partnership for Advanced Computing. I.H. Tuncer acknowledges the support of a visiting fellowship from the Rector of UNSW@ADFA for part of this work.

\section{References}

1. J. Young, J.C.S. Lai: Oscillation Frequency and Amplitude Effects on the Wake of a Plunging Airfoil. AIAA Journal (2004) (in press)

2. I.E. Garrick: Propulsion of a Flapping and Oscillating Airfoil. NACA Report 567 (1937)

3. J. Young, J.C.S. Lai: Thrust and Lift Dependence of a Plunging Airfoil on Oscillation Frequency and Amplitude. In: Computational Fluid Dynamics 2002 ed by S. Armfield, P. Morgan, K. Srinivas (Springer, Berlin Heidelberg New York 2002) pp 273-278

4. J.M. Anderson, K. Streitlien, D.S. Barrett, M.S. Triantafyllou: Journal of Fluid Mechanics 360 (1998) pp 41-72

5. I.H. Tuncer, M.F. Platzer: Journal of Aircraft 37, 3 (2000) pp 514-520

6. K. Isogai, Y. Shinmoto, Y. Watanabe: AIAA Journal 37, 10 (1999) pp 11451151

7. I.H. Tuncer, M. Kaya: Optimization of Flapping Airfoils For Maximum Thrust and Propulsive Efficiency. In: 3rd International Conference on Advanced Engineering Design, Prague, Czech Republic, June 1-5 2003

\begin{tabular}{|l|c|c|c|c|c|}
\hline Case & $S t$ & $k$ & $\theta_{0}$ & $\phi$ & $\eta$ \\
\hline Turbulent, $\phi$ fixed & 0.244 & 0.97 & $21.1^{\circ}$ & $75.0^{\circ}$ & 0.722 \\
\hline Turbulent, $\phi$ fixed & 0.198 & 0.80 & $16.1^{\circ}$ & $90.0^{\circ}$ & 0.705 \\
\hline Turbulent, $\phi$ optimized & 0.252 & 1.00 & $21.9^{\circ}$ & $78.6^{\circ}$ & 0.726 \\
\hline Laminar, $\phi$ fixed & 0.280 & 1.10 & $24.5^{\circ}$ & $75.0^{\circ}$ & 0.598 \\
\hline Laminar, $\phi$ fixed & 0.287 & 1.12 & $25.0^{\circ}$ & $90.0^{\circ}$ & 0.636 \\
\hline Laminar, $\phi$ optimized & 0.258 & 1.02 & $22.5^{\circ}$ & $83.3^{\circ}$ & 0.643 \\
\hline
\end{tabular}

Table 1. Optimization results, $R e=40,000, \alpha_{0}=15^{\circ}$, pivot at $1 / 3$ chord, $h=$ 0.75 . 

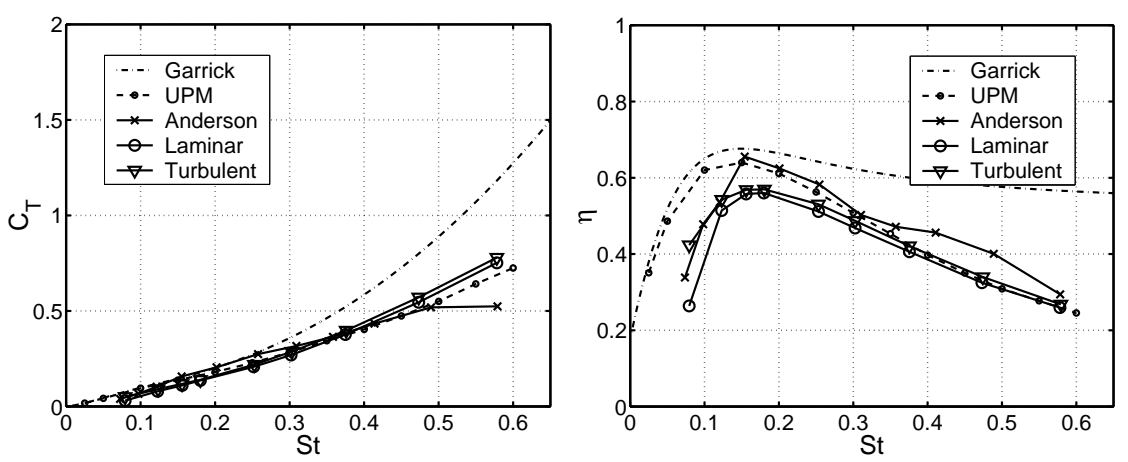

Fig. 1. Mean thrust coefficient and propulsive efficiency, $R e=40,000, \alpha_{0}=15^{\circ}$, pivot at $1 / 3$ chord, $h=0.25, \phi=90^{\circ}$.
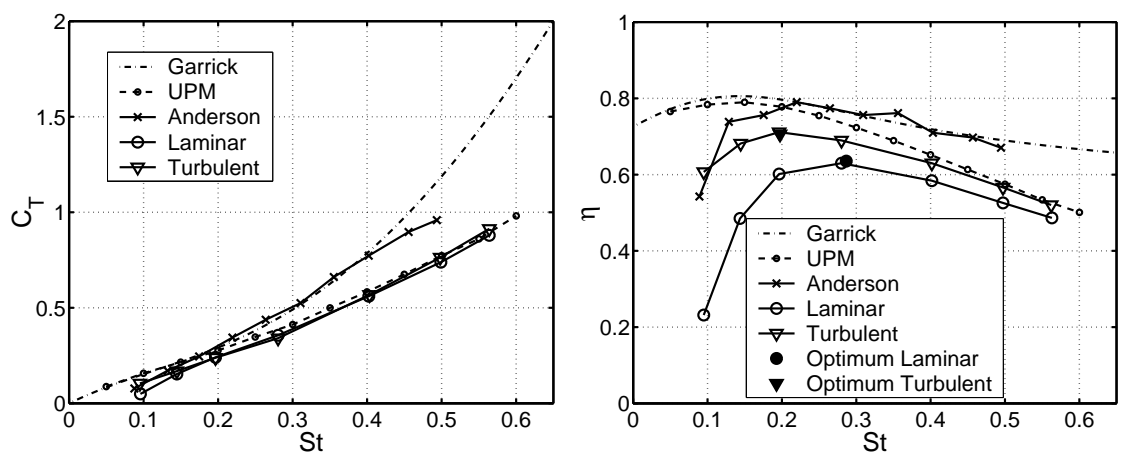

Fig. 2. Mean thrust coefficient and propulsive efficiency, $R e=40,000, \alpha_{0}=15^{\circ}$, pivot at $1 / 3$ chord, $h=0.75, \phi=90^{\circ}$.
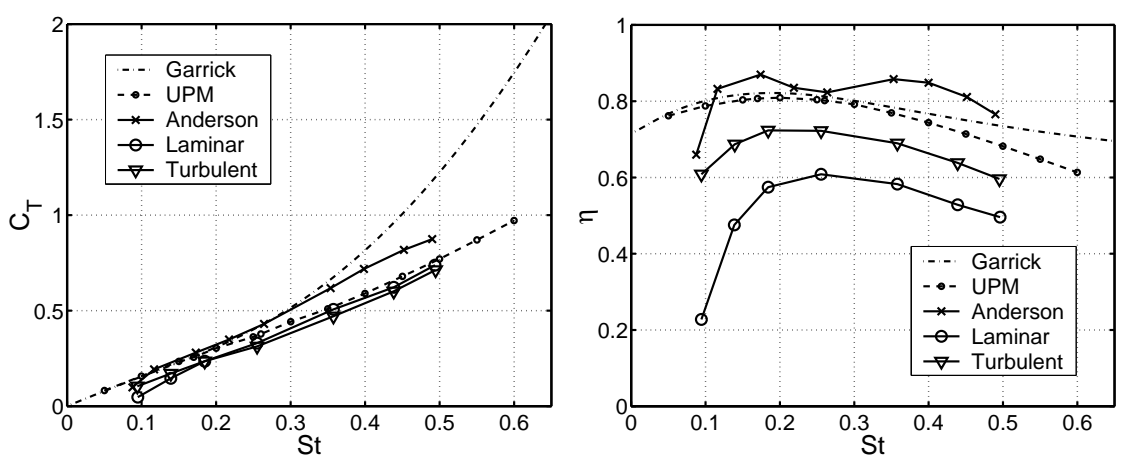

Fig. 3. Mean thrust coefficient and propulsive efficiency, $R e=40,000, \alpha_{0}=15^{\circ}$, pivot at $1 / 3$ chord, $h=0.75, \phi=75^{\circ}$. 

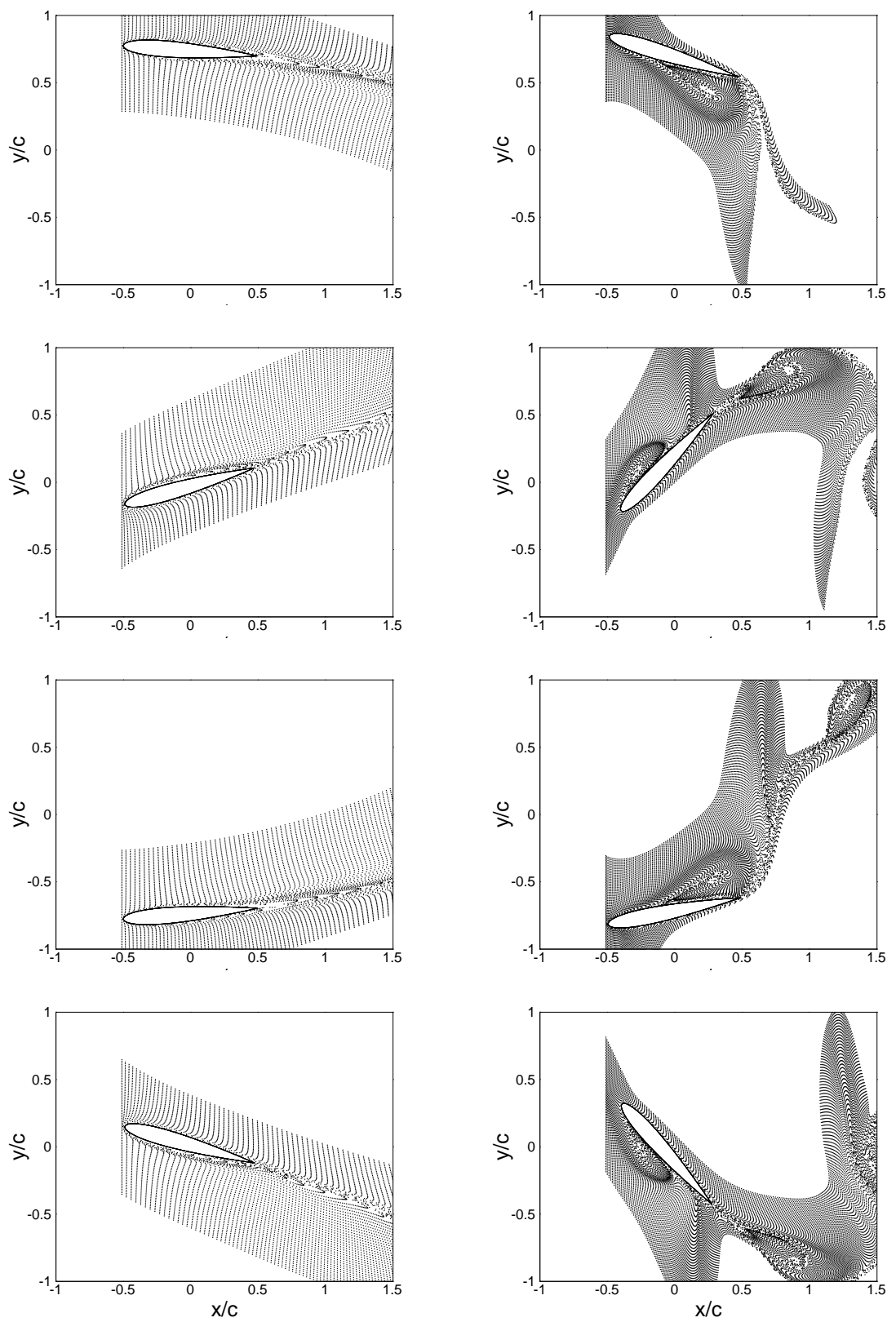

Fig. 4. Particle traces, $\alpha_{0}=15^{\circ}, h=$ $0.75, \phi=75^{\circ}, S t=0.184$

Fig. 5. Particle traces, $\alpha_{0}=15^{\circ}, h=$ $0.75, \phi=75^{\circ}, S t=0.70$ 